\title{
Etude De L'efficacité Du Cashew Nut Shell Liquid (CNSL) De Anacardium Occidentale L. Extrait À Froid Sur Le Contrôle De Anopheles Gambiae S.L Résistant Aux Pyréthrinoïdes
}

\author{
Armand A. Akpo \\ Faculté des Sciences et Techniques, Université d'Abomey-Calavi, Bénin
}

\section{Daniel C. Chougourou}

Ecole polytechnique d'Abomey-Calavi, Département Génie de

l'Environnement, Université d'Abomey-Calavi, Bénin

\section{Armel Djènontin}

Laboratoire Evolution Biodiversité des Arthropodes et Assainissement, Université d'Abomey-Calavi, Bénin

\section{Joseph Dossou}

Département de Nutrition et Sciences Alimentaires, Faculté des Sciences Agronomiques (FSA), Université d'Abomey-Calavi, 01 BP 526 Cotonou, Bénin

\section{Rodrigue Anagonou}

Faculté des Sciences et Techniques, Université d'Abomey-Calavi, Bénin

\section{Martin Akogbéto}

Faculté des Sciences et Techniques, Université d'Abomey-Calavi, Bénin

doi: 10.19044/esj.2017.v13n24p249 URL:http://dx.doi.org/10.19044/esj.2017.v13n24p249

\begin{abstract}
In order to combat the vectors of malaria, it is necessary to know their behavior with regard to the insecticide products envisaged. The present study is aimed at the CNSL efficiency test extracted from the seed hull of Anacardium occidentale L. for the control of pyrethroid-resistant Anopheles gambiae s.l. Sensitivity tests were carried out on the larvae of stages 2, 3 and 4 of An. gambiae of the wild and Kis-kdr strains carrying the Kdr gene. The "Kisumu" strain devoid of resistance gene served as a control. The mortalities were read after 24 hours of exposure. LD50 and DL90 were determined using the log-probit method of dose determination corresponding to a proportion. The high LD50 determined is $0.0042 \mathrm{mg} / \mathrm{ml}$ and corresponds to the LD50 of the larvae of stage 2 of the wild strain. The low LD50 is determined on stage 2 larvae "Kisumu". The high DL90 is determined on stage 4 larvae of the wild strain. The low LD90 determined
\end{abstract}


corresponds to stage 2 larvae "Kisumu". The comparison of the LD between the different strains shows a low sensitivity to CNSL of the larvae of resistant strains compared to the control strain, indicating the influence of the resistance factor kdr on the CNSL efficiency. However, the low-dose CNSL larvicidal activity on the larvae of the kdr-carrying strains provides an alternative advantage in the management of vector resistance to pyrethroids.

Keywords: Larvicidal activity, sensitivity test, resistant An. gambiae, vector control

\section{Résumé}

Pour lutter contre les vecteurs du paludisme, il est nécessaire de connaitre leur comportement vis-à-vis des produits insecticides envisagés. La présente étude vise l'étude de l'efficacité du CNSL extrait à froid de la coque des graines de Anacardium occidentale L. sur le contrôle de Anopheles gambiae s.l résistant aux pyréthrinoïdes. Des tests de sensibilité ont été réalisés sur les larves des stades 2, 3 et 4 de An. gambiae des souches sauvage et Kis-kdr porteuses de gène Kdr. La souche «Kisumu» dépourvue de gène de résistance a servi de témoin. Les mortalités ont été lues après 24 heures d'exposition. Les DL50 et DL90 ont été déterminées suivant la méthode log-probit de détermination de la dose correspondant à une proportion. La forte DL50 déterminée est de $0,0042 \mathrm{mg} / \mathrm{ml}$ et correspond à la DL50 des larves de stade 2 de la souche sauvage. La faible DL50 est déterminée sur les larves de stade 2 «Kisumu».La forte DL90 est déterminée sur des larves de stade 4 de la souche sauvage. La faible DL90 déterminée correspond aux larves de stade 2 «Kisumu». La comparaison des DL entre les différentes souches montre une faible sensibilité au CNSL des larves de souches résistantes par rapport à la souche témoin, ce qui indique l'influence du gène de résistance $\mathrm{Kdr}$ sur l'efficacité du CNSL. Toutefois, l'activité larvicide du CNSL à faible dose sur les larves des souches porteuses du gène $\mathrm{Kdr}$ offre un avantage alternatif dans la gestion de la résistance des vecteurs aux pyréthrinoïdes.

Mots clés: Activité larvicide, test de sensibilité, An.gambiae résistant, lutte antivectorielle

\section{Introduction}

La pharmaco-résistance du Plasmodium falciparum remarquée suite au traitement des différentes formes (simple ou grave) du paludisme avec la propagation de la résistance des vecteurs aux pyréthrinoïdes (Akogbéto et Yakoubou, 1999) et aux carbamates (Djogbénou et al. 2010 ; Aïkpon et al.,2013) constituent un véritable facteur ayant limité la réussite des objectifs de l'OMS (Organisation Mondiale de Santé) vis-à-vis de cette parasitose. En 
effet, les moustiquaires imprégnées d'insecticides à longue durée d'action et les pulvérisations intra domiciliaires sont les principaux moyens de lutte utilisés par les programmes nationaux dans la lutte contre les vecteurs de maladies. A cet effet, quatre classes d'insecticides sont recommandées par l'OMS. Il s'agit des carbamates, les organochlorés, les organophosphorés et les pyréthrinoïdes. L'utilisation de ces moyens de lutte antivectorielle a permis entre temps de réduire, non seulement la transmission du parasite mais aussi la nuisance infligée par les piqûres des moustiques (Carnevale et al., 1988; Magesa et al., 1991; Beach et al., 1993; Akogbéto et al., 1995 ; Akogbéto et al., 2010 ; Mashauri et al., 2013). Toutefois, il a été révélé suite à l'usage des produits chimiques dans la lutte contre le paludisme, une sélection et une expansion remarquables de la résistance des vecteurs aux insecticides dans le temps, dans l'espace et de génération en génération (Hamon, 1963a ; Elissa et al., 1993; Vulule et al., 1994 ; Akogbéto et Yakoubou, 1999; Chandre et al., 1999; Diabateet al., 2002b; Corbel et al., 2007; Yadouléton et al., 2010). Au Bénin en particulier, les travaux réalisés dans ce domaine ont montré que la fréquence allélique du gène de résistance $\mathrm{kdr}$ qui confère la résistance croisée au DDT et aux pyréthrinoïdes est supérieure à 75\% (Gnanguénon et al., 2015). D’autres études réalisées au Bénin sur la dynamique spatiale de la propagation de la résistance aux insecticides ont confirmé cette résistance et son évolution dans le temps et dans l'espace (Djogbénou et al., 2010; Sovi et al., 2013; Djègbè et al.,2014; Gnanguénon et al., 2015), ce qui incrimine l'efficacité des moustiquaires imprégnées de pyréthrinoïdes prouvée par N'Guéssan et al. (2007) en case expérimentale. Les différents mécanismes de résistance développés par les moustiques vis-à-vis des insecticides chimiques et connus sont: (1)- la modification de la cible des pyréthrinoïdes et des organochlorés sur le codon 1014 du canal sodium voltage dépendant (Martinez-Torres et al., 1998 ; Ranson et al., 2000); (2)- la résistance cuticulaire et (3)- la résistance métabolique. Ce dernier est caractérisé généralement par une surproduction des enzymes de détoxication susceptibles de détruire l'insecticide avant son contact avec la cible. Les travaux réalisés par Agossa et al. (2015) sur les mécanismes biochimiques de la résistance de An. gambiae ont montré une activité significativement élevée de certaines enzymes de détoxification telles que les oxydases, les estérases ( $\alpha$ et $\beta$ estérases), les glutathion-Stransférases. La présence, généralement conjointe de ces différentes formes de résistance à forte fréquence dans les populations des vecteurs et leur expansion considérableau Bénin sont bien liées à l'usage massif, continu et fréquent d'un même insecticide ou des insecticides étroitement liés, non seulement en santé publique, mais aussi et surtout en agriculture (Akogbéto et al., 2006; Chouaibou et al., 2008; Yadouléton et al., 2010), ce qui exerce une pression sélective sur les larves et les adultes des vecteurs. La gestion de 
la résistance des vecteurs du paludisme aux insecticides doit alors être partie intégrante de la stratégie de lutte intégrée contre le paludisme. L'une des mesures alternatives prônée est l'usage des outils et méthodes de lutte non chimiques pour autant qu'ils contribuent efficacement à lutter contre les vecteurs résistants. C'est pourquoi nous nous sommes intéressés aux biopesticides obtenus à partir des graines de Anacardium occidentale L. pour le contrôle de An.gambiae s.s résistant aux pyréthrinoïdes. Cette étude a permis d'évaluer l'efficacité (propriétés larvicides) du CNSL contre les populations résistantes et sensibles des vecteurs du paludisme au Bénin.

\section{Matériel et méthodes}

\section{Zone d'étude}

L'étude a été menée entre novembre 2014 et décembre 2015 dans la ville de Cotonou, département du Littoral (Bénin) précisément dans les localités de Zogbo, Ladji, Fifadji, Gbèdjromèdé, Houéyiho 1, Houéyiho 2, Minontchou, Yénawa et Sourou-Léré (figure 1). La ville de Cotonou a des caractéristiques géographiques qui sont favorables à la prolifération des moustiques. Elle présente une plaine sableuse sans relief, avec une nappe phréatique peu profonde et affleurant le sol (Akogbéto, 1992). Cette absence de relief, associée à un mauvais drainage des eaux, provoque des inondations pendant les saisons pluvieuses et favorise la multiplication des gites des Culicidae dont An. gambiae (Akogbéto et Yakoubou, 1999; Akogbéto, 1992). Par ailleurs, la ville de Cotonou est marquée par l'aménagement des terres et périmètres maraîchers, qui créent de véritables gîtes de Culicidae dont An.gambiae, le principal vecteur du paludisme (Akogbéto, 1992; Akogbéto, 1995). Cotonou abrite aussi le plus grand port du pays qui importe et exporte des conteneurs dans lesquels peuvent se cacher des moustiques venant d'autres horizons. Ces caractéristiques de la ville créent à ses habitants une nuisance culicidienne très forte. En effet, on y rencontre des flaques d'eau, des marécages temporaires et permanents, des pneus abandonnés ou stockés dans des garages à ciel ouverts...etc. C'est l'une des villes les plus affectées par le paludisme au Bénin (MS, 2013). 


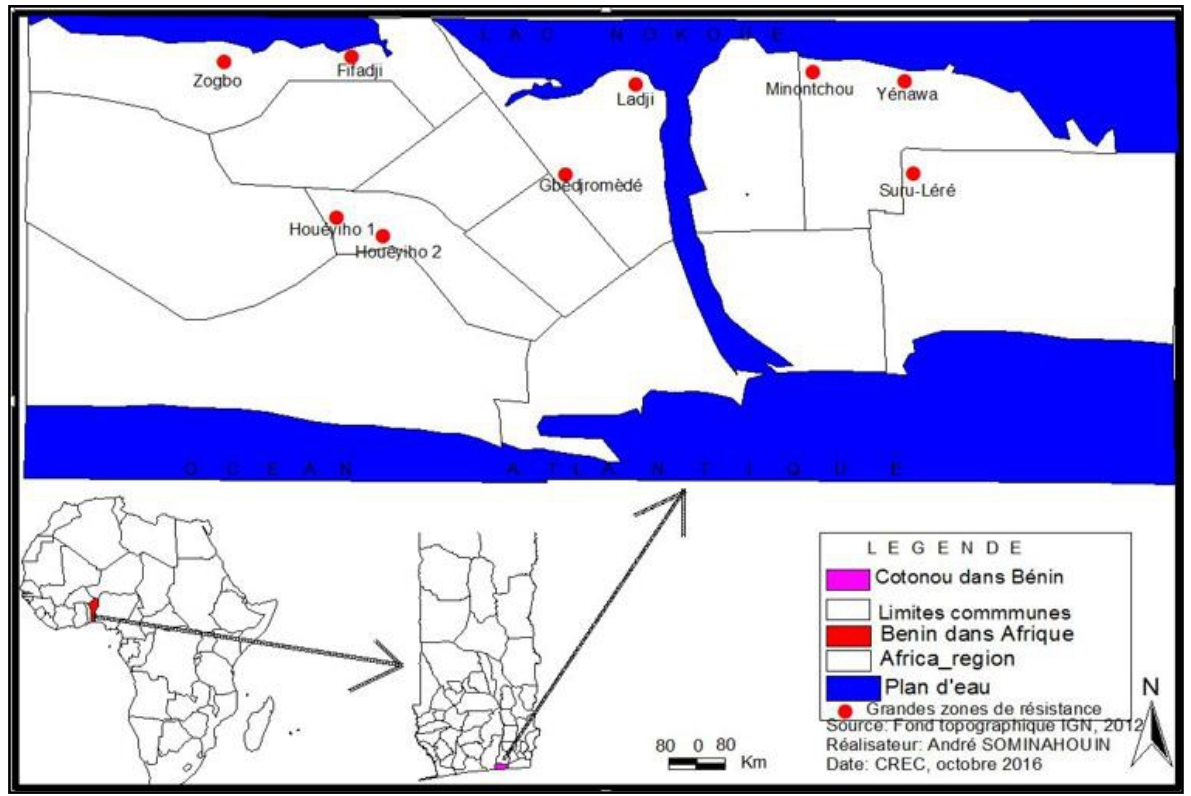

Figure 1: Carte montrant la zone d'étude

\section{Matériel biologique \\ Matériel animal}

Les tests ont été réalisés sur les larves de An. gambiae s.l. Trois souches ont été utilisées. Il s'agit de la souche de référence sensible «Kisumu» de génotype SS, la souche Kis-kdr de génotype RR, résistante aux pyréthrinoïdes et aux organochlorés puis la souche sauvage caractérisée par une fréquence allélique élevée $(>80 \%)$ du gène de résistance $\mathrm{Kdr}$. Les souches Kisumu et Kis-kdr sont élevées à l'insectarium du Centre de Recherche Entomologique de Cotonou (CREC). Les anophèles femelles adultes des deux souches sont conditionnés à une température de $26^{\circ} \mathrm{C} \pm 2{ }^{\circ} \mathrm{C}$ grâce à un climatiseur qui fonctionne en permanence. La ponte des moustiques est assurée par des repas sanguins. Pour cela, des femelles âgées de trois à cinq jours sont gorgées pendant une vingtaine de minutes grâce à des lapins immobilisés sur des cages de moustiques par un dispositif élaboré en conséquence. Après le repas sanguin, les femelles gorgées sont continuellement nourries au jus de miel à $10 \%$ dans du coton déposé sur le tulle moustiquaire des cages. Cela sert de nourriture pour les femelles gravides. Après la ponte, les œufs des différentes souches sont récoltés quotidiennement en retirant les pondoirs des cages. Les pondoirs sont systématiquement renouvelés après chaque retrait. Les œufs recueillis sont mis en eau (l'eau de robinet) dans des bacs en vue de leur éclosion. La salle d'élevage des larves est conditionnée à une plage de températures de $29^{\circ} \mathrm{C}$ à $32^{\circ} \mathrm{C}$ favorables au développement des stades pré imaginaux des moustiques et ceci à l'aide d'un radiateur de chauffage qui diffuse des calories par rayonnement. Les larves obtenues après éclosion sont nourries de croquette 
de chat dès le premier stade. L'eau d'élevage est renouvelée après 72 heures afin de garantir une bonne croissance des larves. Cela a permis d'obtenir des larves de stades 1, 2, 3, 4 et les nymphes des différentes souches. Les larves et nymphes des moustiques sauvages sont récoltées directement dans les gîtes des sites d'étude aux moyens de louches, de pipettes Pasteur, de bocaux et de filtres à mailles serrées pouvant retenir les larves de différents stades.

\section{Matériel végétal}

La matière végétale sujette de notre étude a été prélevée sur des plantes issues de la flore béninoise (tableau 1).

Tableau 1: Espèce végétale et organe utilisés

\begin{tabular}{|c|c|c|c|}
\hline Espèce végétale & Partie utilisée & Nature des extraits & Méthodes \\
\hline $\begin{array}{c}\text { Anacardium } \\
\text { occidentale } L .\end{array}$ & Coque des graines & CNSL à froid & $\begin{array}{c}\text { Coque brute, fragilisée } \\
\text { sans traitement } \\
\text { préalable et soumise à } \\
\text { l'extraction par presse } \\
\text { à vis }\end{array}$ \\
\hline
\end{tabular}

CNSL : Cashew Nut Shell Liquid

\section{Description de la méthode d'extraction à froid du CNSL}

L'extraction du CNSL a été réalisée au laboratoire (Laboratoire de Bio ingénierie et de Production Alimentaire de la Faculté des Sciences Agronomique, Université d'Abomey-Calavi) à froid avec presse des coques brutes des noix de Anacardium occidentale fragilisées. La fragilisation des noix se fait à la vapeur grâce à un système constitué d'une source de chaleur, chauffant l'eau contenue dans une chaudière jusqu'à une température entrainant une pression de 10 bars. Cette pression correspond à une température de $180^{\circ} \mathrm{C}$. La chaudière est reliée à un autoclave (contenant les noix à fragiliser) par un conduit muni d'une vanne qui s'ouvre lorsque la pression atteint les 10 bars.

Tests larvaires : Etude de l'efficacité du CNSL extrait à froid sur les larves de An. gambiae s.s résistants aux pyréthrinoïdes

Les tests larvaires ont été réalisés au CREC sur les larves des stades 2,3 et 4 des souches sensibles «Kisumu» et résistantes (Kis-Kdr de laboratoire et sauvages) conformément au protocole préconisé par l'Organisation Mondiale de la Santé(Abbott, 1925; OMS, 1963, 2004\& 2014). Les larves sont exposées à des gammes de concentrations de la matière insecticide dans des gobelets contenant chacun 20 larves. Pour chaque stade larvaire des différentes souches de moustiques, des concentrations formulées à partir du CNLS ont été préparées en plus des témoins à raison de cinq répétitions par concentration de l'extrait. Les concentrations sub-létales ont été choisies de manière à obtenir des taux de 
mortalité pouvant varier de $0 \%$ à $100 \%$. Les larves mortes après 24 heures d'exposition aux produits insecticides formulés sont dénombrées suivant chaque dose appliquée. Les résultats sont exprimés en pourcentage de mortalité. Ce qui est déterminé en additionnant les nombres de larves mortes dans les réplications de chaque concentration du test d'exposition par rapport au nombre total de larves de moustique exposées. Les mortalités dans les lots témoins sont également déterminées de la même manière selon la formule:

Mortalité au cours du test

$$
\begin{aligned}
& =\frac{\text { Nombre total de larves mortes }}{\text { Taille totale de l'échantillon }- \text { Nombre de nymphes }} \\
& \times 100
\end{aligned}
$$

Lorsque le taux de mortalité enregistré au niveau des témoins est supérieur à $20 \%$, le test est invalide. Si la mortalité des témoins est supérieure à $5 \%$ et inférieur à $20 \%$, la mortalité observée est corrigée par la formule d'Abbott :

$$
\text { Mortalité corrigée }=\frac{\% \text { mortalité lors du test }-\% \text { mortalité témoin }}{100-\% \text { mortalité témoin }}
$$

Si la mortalité au niveau du témoin est inférieure à $5 \%$ elle peut être ignorée et bien, aucune correction n'est nécessaire.

Pour la détermination des DL-50 et DL-90au CNSL sur les différents stades larvaires, le logiciel R Core Team (Version 3.3.1-2016) a été utilisé. La méthode utilisée est celle de log-probit de détermination de la dose correspondant à une proportion donnée.

\section{Formulation du CNSL extrait à froid.}

Une solution stock du CNSL dans l'éthanol a été préparée. A partir de cette solution mère, une série de concentrations a été réalisée en vue de varier les doses pouvant induire des mortalités allant de $0 \%$ à $100 \%$. La dispersion de $30 \mathrm{mg}$ de baume nécessite $\frac{1}{10} \mathrm{ml}$ d'éthanol dans un volume de $30 \mathrm{ml}$ d'eau distillée. La solution stock a été donc préparée en dissolvant 200 $\mathrm{mg}$ de baume dans $66.10^{-2} \mathrm{ml}$ d'éthanol pour un volume total de $200 \mathrm{ml}$ d'eau distillée.

\section{Résultats}

Le CNSL extrait à froid a montré un effet larvicide sur les larves de stade 2, 3 et 4 des souches «Kisumu», Kis-kdr et sauvages. Les mortalités enregistrées sont comparées à chaque stade pour les trois souches testées. 


\section{Efficacité du CNSL extrait à froid sur les larves de stade 2 des souches sensible Kisumu, Kis-kdr et sauvage de An. gambiae s.l.}

Après avoir exposé les larves de stade 2 des souches sensible Kisumu, Kis-kdr et sauvage aux différentes concentrations du CNSL, le taux de mortalité enregistré varie en fonction des concentrations (figure 2). Les larves de stade 2 des trois souches ont une sensibilité différentielle vis-à-vis du CNSL extrait à froid. Pour une forte dose de $0,01 \mathrm{mg} / \mathrm{ml}$, on enregistre une mortalité de $100 \%$ des larves des trois souches. En dessous de cette dose, les mortalités enregistrées au niveau des larves des populations sauvages diminuent progressivement et atteignent une valeur inférieure à $80 \%$ à une dose de $0,005 \mathrm{mg} / \mathrm{ml}$. Cependant, on enregistre toujours une mortalité de $100 \%$ des larves des souches Kis-kdr et Kisumu à cette concentration de $0,005 \mathrm{mg} / \mathrm{ml}$. La faible dose entrainant $100 \%$ de mortalité de la souche «Kisumu» est $0,00375 \mathrm{mg} / \mathrm{ml}$. Elle est de $0,005 \mathrm{mg} / \mathrm{ml}$ pour la souche Kis$\mathrm{kdr}$ alors que la mortalité des larves de la souche sauvage atteint le plafond des $100 \%$ à partir de la concentration de $0,01 \mathrm{mg} / \mathrm{ml}$. La mortalité des larves sauvages atteint les $50 \%$ à une dose inférieure à $0,0025 \mathrm{mg} / \mathrm{ml}$. Concernant les larves de la souche «Kisumu», les 50\% de mortalité sont enregistrés à une dose inférieure à $0,001 \mathrm{mg} / \mathrm{ml}$. La mortalité des larves des populations sauvages diminue et tend à s'annuler à une dose de $0,001 \mathrm{mg} / \mathrm{ml}$ alors que cette dose entraine une mortalité supérieure à $20 \%$ des larves Kis-kdr et $70 \%$ des larves de la souche sensible «Kisumu».Il ressort de ces observations que les larves de stade 2 sauvages sont moins sensibles au CNSL extrait à froid que les larves de la souche Kis-kdr qui sont aussi moins sensibles que la souche «Kisumu».

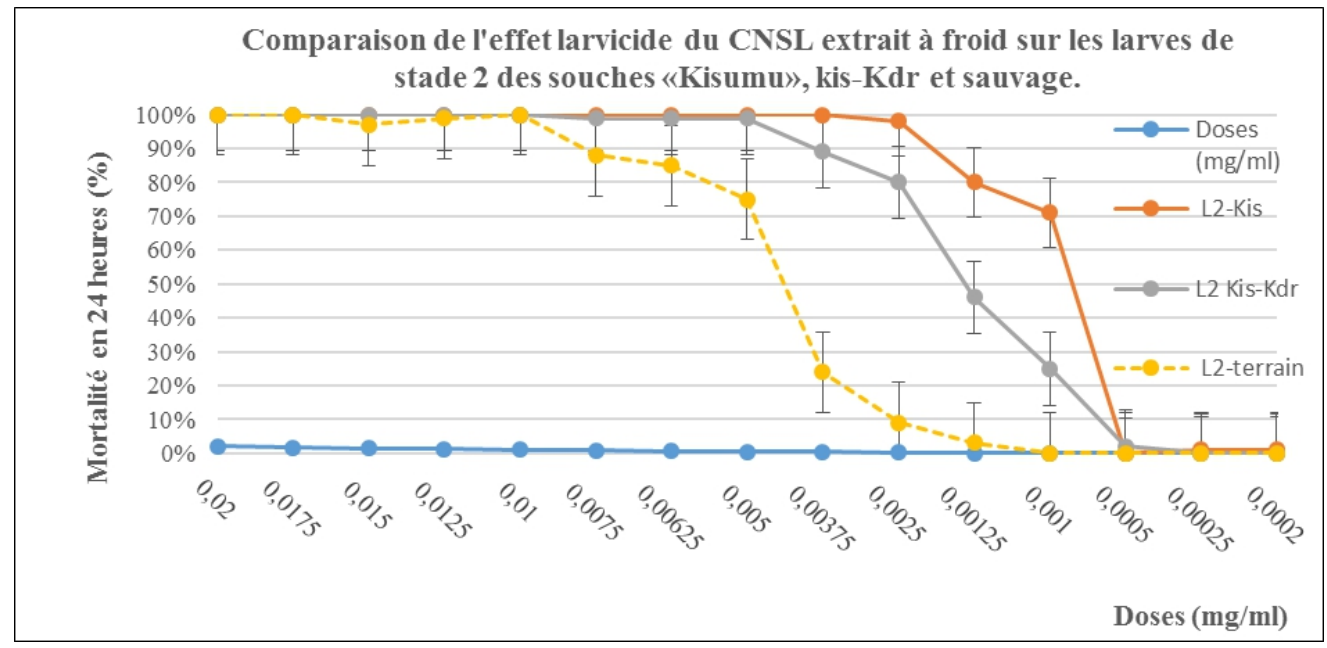

Figure 2: Comparaison de l'effet larvicide du CNSL extrait à froid sur les larves de stade 2 des souches «Kisumu», Kis-kdr et sauvage. 


\section{Efficacité du CNSL extrait à froid sur les larves de stade 3 des souches sensible «Kisumu», Kis-kdr et sauvage de An.gambiaes.l.}

La figure 3montre l'évolution des pourcentages de mortalité des larves de stade 3 des trois populations de moustiques étudiées en fonction des doses testées. Les mortalités enregistrées sur les larves de stade 3 des souches «Kisumu», Kis-kdr et sauvage varient en fonction des doses formulées. A $0,01 \mathrm{mg} / \mathrm{ml}$, on note $100 \%$ de mortalité au niveau des trois souches testées. En dessous de cette dose on observe une régression progressive des mortalités de la souche sauvage alors qu'une similarité a été notée sur les souches Kis-kdr et «Kisumu» aux doses allant de 0,01 à $0,00625 \mathrm{mg} / \mathrm{ml}$. Cependant, on note à ces doses que les larves de souche sauvage sont moins sensibles par rapport aux souches Kis-kdr et «Kisumu». En dessous de la dose $0,00625 \mathrm{mg} / \mathrm{ml}$, le taux de mortalité des trois souches diminue progressivement avec une similarité notée entre les doses allant de 0,005 à $0,00375 \mathrm{mg} / \mathrm{ml}$ sur les souches «Kisumu» et sauvage. En dessous de ces doses, les souches sauvages, Kis-kdr et «Kisumu» présentent une mortalité diminuant progressivement et qui s'annule à $0,00125 \mathrm{mg} / \mathrm{ml}$. Les larves de souche sauvage présentent une sensibilité faible par rapport aux souches «Kisumu» et Kis-kdr aux doses allant de 0,0125 à $0,005 \mathrm{mg} / \mathrm{ml}$ puis aux doses inférieures à $0,00375 \mathrm{mg} / \mathrm{ml}$ et supérieures à $0,00125 \mathrm{mg} / \mathrm{ml}$. Toutefois les larves de souche sauvage présentent un caractère moins sensible avec des mortalités plus faibles que celles enregistrées au niveau de la souche Kis-kdr pour les doses de 0,01 à $0,00125 \mathrm{mg} / \mathrm{ml}$.

Ces résultats enregistrés montrent que les larves de stade 3 des populations de An. gambiae sauvages sont moins sensibles au CNSL extrait à froid par rapport à la souche Kis-kdr.

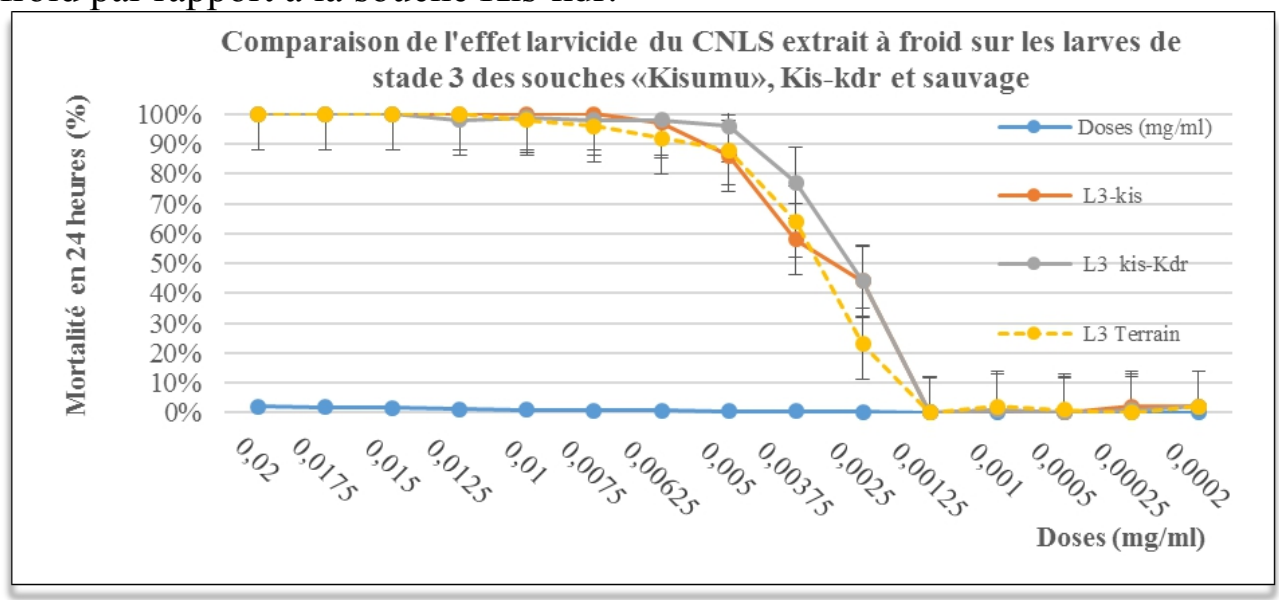

Figure 3: Comparaison de l'effet larvicide du CNSL extrait à froid sur larves de stade 3 des souches «Kisumu», Kis-kdr et sauvage. 


\section{Efficacité du CNSL extrait à froid sur les larves de stade 4 des trois souches de An. gambiae s.l étudiées.}

La figure 4montre l'évolution des pourcentages de mortalités des larves de stade 4 des trois souches étudiées en fonction des doses testées. Cette figure indique une mortalité de $100 \%$ des larves de stade 4 de la souche sauvage à la dose limite de $0,015 \mathrm{mg} / \mathrm{ml}$. En dessous de cette dose, la mortalité de ces larves régresse progressivement et atteint les valeurs limites de $70 \%$ correspondant à $0,0075 \mathrm{mg} / \mathrm{ml}$ qui est la limite inférieure induisant $100 \%$ de mortalité des larves de la souche «Kisumu». En dessous de cette dose, on observe une faible pente dans la représentation graphique (figure 4) exprimant la variation du taux de mortalité des larves de la souche «Kisumu» et celles des populations de souche sauvage. Cette faible pente enregistrée traduit une faible activité larvicide du CNSL aux doses allant de 0,0075 à $0,005 \mathrm{mg} / \mathrm{ml}$. La mortalité dépasse les $50 \%$ sur les souches porteuses du gène $\mathrm{Kdr}$ et la souche témoin «Kisumu» à partir de la dose de $0,00375 \mathrm{mg} / \mathrm{ml}$ et s'annule d'abord au niveau de la souche sauvage avec les doses inférieures à $0,00125 \mathrm{mg} / \mathrm{ml}$. Chez les souches kis-kdr et «Kisumu», la mortalité devient nulle avec les doses inférieures ou égales à $0,0005 \mathrm{mg} / \mathrm{ml}$. Avec les doses allant de 0,015 à $0,00125 \mathrm{mg} / \mathrm{ml}$, les larves des populations sauvages manifestent une faible sensibilité au CNSL extrait à froid par rapport aux souches Kis-kdr et «Kisumu». De $0,005 \mathrm{mg} / \mathrm{ml}$ aux limites supérieures de $0,00125 \mathrm{mg} / \mathrm{ml}$, la souche Kis-kdr apparait moins sensible que la souche «Kisumu». A 0,00125 mg/ml, la souche Kisumu parait moins sensible que la souche Kis-kdr. En dessous de cette dose jusqu'à $0,0005 \mathrm{mg} / \mathrm{ml}$, les souches Kis-kdr et «Kisumu» présentent une faible sensibilité avec une activité larvicide plus forte du CNSL sur la souche «Kisumu».

Il ressort donc de ces résultats que le CNSL présente une activité larvicide intéressante sur les larves de stade 4 des souches sauvages, Kis-Kdr et «Kisumu». Cependant, les souches sauvage et Kis-kdr apparaissent moins sensibles que la souche de référence sensible «Kisumu».

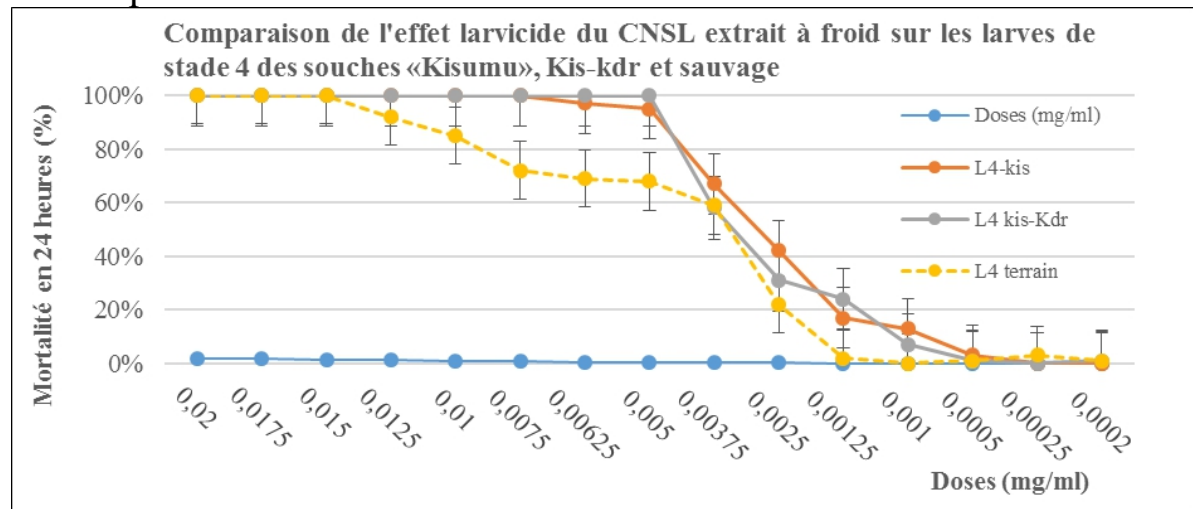

Figure 4: Comparaison de l'effet larvicide du CNSL extrait à froid sur les larves de stade 4 des souches «Kisumu», Kis-kdr et sauvage 
Comparaison des DL50 et DL90 du CNSL sur les larves de stade 2, 3 et 4 de $A$ n. gambiaedes souches «Kisumu», Kis-kdr et sauvage

A l'issu de nos travaux, nous avons comparé les DL50 et DL90 du CNSL des stades des différentes populations de moustiques. La représentation graphique ci-dessous (figure 5) regroupe l'ensemble des DL50 et DL90 des différents stades des différentes populations. Le CNSL extrait à froid a une activité larvicide sur les larves de moustique de souches sensible et celles résistant aux pyréthrinoïdes. La détermination des DL50 et DL90 du CNSL sur les larves des stades 2, 3 et 4 des souches «Kisumu», Kis-kdr et sauvage a montré une activité larvicide à faible dose et variant d'une souche à une autre. La forte DL50 enregistrée est de $0,0042 \mathrm{mg} / \mathrm{ml}$ et correspond à la DL50 des larves de stade 2 des populations de moustiques sauvages. La faible DL50 enregistrée est de $0,0009 \mathrm{mg} / \mathrm{ml}$ et correspond à la DL50 des larves de stade 2 «Kisumu». Concernant la DL90, la plus forte enregistrée est de $0,0107 \mathrm{mg} / \mathrm{ml}$ et correspond à la DL90 des larves de stade 4 des souches sauvages. La faible DL90 est $0,0015 \mathrm{mg} / \mathrm{ml}$ et correspond à la DL90 des larves de la souche «Kisumu» au stade 2. Aux stades 2 et 4, les DL50 déterminées ont révélé que les larves des populations de moustiques sauvages sont moins sensibles au CNSL que la souche Kis-kdr qui est aussi moins sensible que la souche «Kisumu». Au stade 3, les larves Kis-kdr apparaissent moins sensibles. Les DL90 montrent aux stades 2, 3 et 4 une faible sensibilité des larves de la souche sauvage au CNSL comparativement aux larves de la souche Kis-kdr, lesquelles sont moins sensibles que la souche «Kisumu». Il apparait donc à travers la variation des doses létales selon les souches testées porteuses ou non du facteur de résistance kdr que la présence de ce facteur de résistance influence la sensibilité des larves de $A n$. gambiae s.sau CNSL extrait à froid.

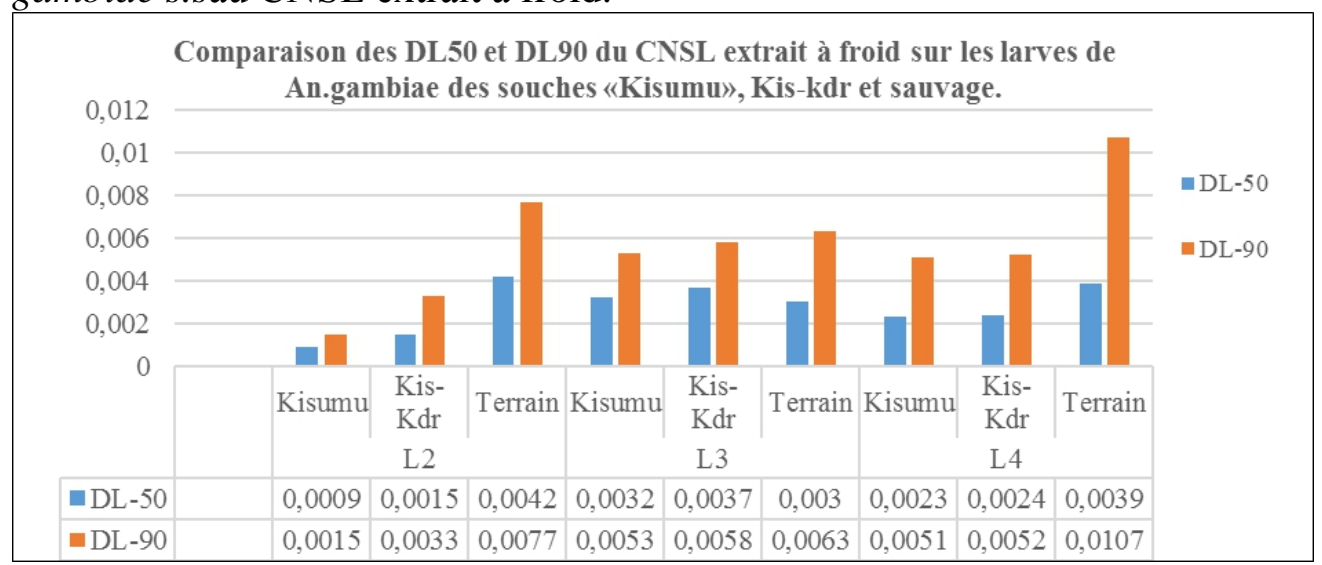

Figure 5:Comparaison des DL50 etDL90 du CNSL extrait à froid sur les larves de An. gambiae s.s des souches «Kisumu», Kis-kdr et sauvage. 


\section{Discussion}

L'intégration d'un insecticide nouveau pour la lutte contre les vecteurs dans le contexte de la gestion de la résistance nécessite la bonne connaissance de l'effet insecticide du produit envisagé vis-à-vis des vecteurs cibles. La présente étude est consacrée à l'étude de la sensibilité des larves de An. gambiae résistant aux pyréthrinoïdes.

La forte sensibilité à faible dose au CNSL des larves de souche pourvue de l'allèle de résistance $\mathrm{Kdr}$ constitue une bonne nouvelle dans le cadre de la mise en œuvre des mesures alternatives pour la gestion de la résistance des vecteurs en santé humaine. Toutefois, l'activité larvicide du CNSL à faible dose sur les larves des populations sensibles pourrait par ailleurs engendrer une pression sélective des vecteurs cibles, donc une nouvelle émergence des vecteurs pouvant tolérer des doses létales en ce sens que la sélection des gènes de résistance est généralement induite avec le contact des doses faibles de polluants (David et al. 2006; Nkya et al.,2014; Antonio-Nkondjio et al.,2014; Poupardin et al.,2008; Raghavendra et al.,2010). Il faut noter aussi sur cet aspect de sélection éventuelle des vecteurs résistant que très peu d'étude ont prouvé jusque-là et de façon tangible les risques de résistance liés à l'usage des biopesticides.

La sensibilité différentielle enregistrée sur les souches sauvage et Kis-kdr toutes porteuses de du gène $\mathrm{kdr}$ par rapport à la souche de référence «Kisumu» indique bien l'influence de l'expression du gène kdr sur la sensibilité au CNSL des larves. Une zone de forte fréquence des allèles du gène kdr (Djogbénou et al., 2010; Sovi et al., 2013; Djègbè et al., 2014; Gnanguénon et al., 2015). La faible sensibilité de ces larves issues des populations sauvages vis-à-vis du CNSL par rapport à la souche Kis-kdr caractérisée par le portage d'un seul gène de résistance nous a amené à envisager l'implication d'autres mécanismes de résistance intrinsèques susceptibles d'influencer l'efficacité du CNSL sur les vecteurs qui sont déjà résistants aux pyréthrinoïdes. Les milieux de développement des larves de An. gambiae s.l. sauvage seraient donc un facteur déterminant leur comportement vis-à-vis des produits insecticides. En effet les travaux de Agossa, (2016) ont montré la présence, non seulement des particules des insecticides utilisés contre les vecteurs en santé humaine et animale et les ravageurs des cultures, mais aussi des polluants d'origine anthropique qui exercent une pression de sélection sur les larves dans les gîtes naturels. Le développement des larves dans ces gîtes indique une forte adaptation aux facteurs environnementaux. La résistance métabolique peut être envisagée comme moyen utilisé par les larves pour survivre dans leur milieu naturel. Cette forme de résistance est caractérisée par la production d'enzyme de détoxication qui dégrade les molécules pouvant exercer une activité létale sur l'insecte. Et du fait que les larves des moustiques sauvages résistant aux 
pyréthrinoïdes sont moins sensibles au CNSL par rapport à la souche de laboratoire pourvue uniquement du gène $\mathrm{kdr}$, l'étude de l'influence de la résistance métabolique sur l'efficacité du baume mérite d'être vérifiée dans ce contexte. Toutefois, on pourra se réjouir de l'effet biocide du CNSL sur les larves des souches porteuses de gènes $\mathrm{Kdr}$, en envisageant toutes formulations pouvant améliorer son efficacité dans la gestion de la résistance des vecteurs du paludisme, ce qui pourra préserver l'environnement des conséquences liées à l'usage des insecticides chimiques sice produit disposait d'un caractère moins toxique pour l'environnement avec une bonne innocuité.

\section{Conclusion}

Il ressort de cette étude que le CNSL est efficace dans la gestion des vecteurs résistants. A forte dose, son efficacité est plus renforcée. Le CNSL peut être un bon candidat alternatif dans la lutte anti-larvaire. Cependant, nous suggérons pour l'élaboration des formulations pouvant optimiser son efficacité d'autres études comme celles d'impact environnemental, l'efficacité du baume dans les conditions naturelles. Les DL50 et DL90 des différents stades larvaires des souches testées ont montré que les larves des populations sauvages et kis-kdr sont moins sensibles par rapport à celles de la souche «kisumu» témoin. La sensibilité au CNSL des larves est réduite chez les anophèles résistant aux pyréthrinoïdes. Toutefois l'activité larvicide du CNSL à faible dose sur les larves des souches sauvage et kis-kdr offre un avantage dans la lutte contre les vecteurs résistants. Sa mise en œuvre devrait être une lutte respectueuse pour l'environnement et accessible pour les populations exposées aux risques de nuisance des vecteurs et de transmission du paludisme.

\section{References:}

1. Abbott, W.S, $1925^{\prime A} \mathrm{~A}$ method of computing the effectiveness of an insecticide", J. Econ. Entomo. vol. 18, pp. 265-267.

2. AGBIZOUNON A., 2010: Tests d'efficacité d'extraits d'huiles végétales sur Anopheles gambiae Giles et Culex quinquefasciatus Say résistants aux pyréthrinoîdes. Mémoire Ingénieur de conception, EPAC Abomey- Calavi, Bénin. 51pp.

3. Aïkpon R., Agossa F., Ossè R., Oussou O., Aïzoun N., Oké-Agbo F. \& Akogbéto M. 2013 : Bendiocarb resistance in Anopheles gambiae sl populations from Atacoradepartment in Benin, West Africa: a threat for malaria vector control.ParasitVectors 6: 192-198.

4. Akogbeto M, Djouaka R, Gazard D. A., 2006: Screening of pesticide residues in soil and water samples from agricultural settings. Malar $\mathbf{J}$ 2006, 5:22. 
5. Akogbéto M. et Yakoubou S., 1999: Résistance des vecteurs du paludisme vis-à-vis des pyréthrinoïdes utilisés pour l'imprégnation des moustiquaires au Bénin, Afrique de l'Ouest. Bull. Soc. Pathol. Exot, 92(2): 123-130.

6. AKOGBETO M., 1995 : Etude entomologique sur la transmission du paludisme côtier lagunaire: cas d'un village construit sur un lac d'eau saumâtre. Ann. Soc. Belge Med trop. 75, 219-227.

7. Akogbéto M., Chippaux J.-P. \& Coluzzi M. 1992. Le paludisme urbain côtier à Cotonou (République du Bénin).Étude entomologique. Revue d'Epidémiologie etSanté Publique 40: 233-239.

8. Antonio-Nkondjio C., Youmsi-Goupeyou M., Kopya E., TeneFossog B., Njiokou F.,

9. Beach R.F., Ruebush T.K., Sexton J.D., Bright P.L., Hightower A.W., Breman J.G., Mount D.L. \& Oloo A.J. 1993. Effectiveness of Permethrin-Impregnated Bed Netsand Curtains for Malaria Control in a Holoendemic Area of Western Kenya.TheAmerican Journal of Tropical Medicine and Hygiene 49: 290-300.

10. Bourkhiss M., Hnach M., Bourkhiss B., Ouhssine M., Chaouch A., Satrani B., 2009: Effet de séchage sur la teneur et la composition chimique des huiles essentielles de Tetraclinisarticulata (Vahl) Masters. Agrosolutions. 20(1) 44.

11. Carnevale P. 1998. La protection du voyageur contre les piqures d'arthropodesvecteurs.Bull Soc Pathol Exot 91: 474-485.

12. Chandre F., Darrier F., Manga L., Akogbeto M., Faye O., Mouchet J. \& Guillet P. 1999. Status of pyrethroid resistance in Anopheles gambiae sensu lato.Bulletin of theWorld Health Organization 77: 230-234.

13. Cheng, S.S., Liu, J.Y., Tsai, K.H., Chen, W.J., Chang, S.T., 2004: Chemical composition and mosquito larvicidal activity of essential oils from leaves of different Cinnamomumosmophloeum provenances. J. Agric. Food Chem. 524395.

14. Chouaïbou M., Etang J., Brevault T., Nwane P., Hinzoumbé C.K., Mimpfoundi R. \&Simard F. 2008. Dynamics of insecticide resistance in the malaria vector Anopheles gambiae sl from an area of extensive cotton cultivation in Northern Cameroon.Tropical Medicine \& International Health 13: 476-486.

15. Chougourou C. Daniel (1)* ; Dellouh P. Lesline (1), Agbaka Alphonse (1), N'Guessan K. Raphael (2), Gbenou Joachim D. (3), 2012: Toxicité et effets répulsifs de certaines huiles extraites des plantes locales Béninoises sur la mouche domestique Musca domestica L. (Diptera Muscidae). Journal of Applied Biosciences 55: 3953- 3961. 
16. Corbel V., N'guessan R., Brengues C., Chandre F., Djogbenou L., Martin T., Akogbeto M., Hougard J.M. \& Rowland M. 2007. Multiple insecticide resistance mechanisms in Anopheles gambiae and Culex quinquefasciatus from Benin, West Africa.Actatropica 101: 207-216.

17. Costantini C. \& Awono-Ambene P. 2014. Exposure to disinfectants (soap or hydrogenperoxide) increases tolerance to permethrin in Anopheles gambiae populations fromthe city of Yaoundé, Cameroon. Malaria journal 13: 296.

18. David J.-P., Boyer S., Mesneau A., Ball A., Ranson H. \& DauphinVillemant C. 2006.Involvement of cytochrome P450 monooxygenases in the response of mosquito larvae to dietary plant xenobiotics. Insect biochemistry and molecular biology 36: 410-420.

19. David J.-P., Strode C., Vontas J., Nikou D., Vaughan A., Pignatelli P.M., Louis C.,Hemingway J. \& Ranson H. 2005. The Anopheles gambiae detoxification chip: A highly specific microarray to study metabolic-based insecticide resistance in malaria vectors. Proceedings of the National Academy of Sciences of the United States of America 102: 4080-4084.

20. Diabate A., Baldet T., Chandre F., Guiguemde R.T., Brengues C., Guillet P., Hemingway J. \& Hougard J.M. 2002b. First report of the kdr mutation in Anopheles gambiae $\mathrm{M}$ form from Burkina Faso, west Africa.Parassitologia 44: 157-158.

21. Djègbè I., Agossa F.R., Jones C.M., Poupardin R., Cornelie S., Akogbéto M., Ranson H. \& Corbel V. 2014. Molecular characterization of DDT resistance in Anopheles gambiae from Benin. Parasites \& vectors 7: 1-9.

22. Djènontin A., Sahabi B.B. Nicolas M., Djènontin A., Sahabi B., Moiroux N., Henry MC., Boussari O., Chabi J., Osse R., Koudénoukpo S., Corbel V., Akogbéto M. et Chandre F., 2010: Culicidae diversity, malaria transmission and insecticide resistance alleles in malaria vectors in Ouidah-Kpomasse -Tori district from Benin (West Africa): A pre intervention study.3:83, 7p.

23. Djogbénou L., Noel V. \& Agnew P. 2010. Costs of insensitive acetylcholinesterase insecticide resistance for the malaria vector Anopheles gambiae homozygous for the G119S mutation. Malaria Journal 9: 12.

24. Elissa N., Mouchet J., Rivière F., Meunier J.Y. \& Yao K. 1993. Resistance of Anopheles gambiae ss to pyrethroids in Cote d'Ivoire. Annales-Societe Belge De MedecineTropicale, pp. 291-291.

25. Fiacre R. Agossa, Virgile Gnanguenon, Rodrigue Anagonou, Roseric Azondekon, Nazaire Aïzoun, Arthur Sovi1, Frédéric Oké-Agbo, 
Michel Sèzonlin, Martin C. Akogbéto, 2015 : Impact of Insecticide Resistance on the Effectiveness of Pyrethroid-Based MalariaVectors Control Tools in Benin: Decreased Toxicity and Repellent Effect. doi:10.1371/journal. pone. 0145207

26. Gnanguénon V., Agossa F.R., Badirou K., Govoetchan R., Anagonou R., Oke-Agbo F., Azondekon R., AgbanrinYoussouf R., Attolou R., Tokponnon F.T., Aïkpon R., Ossè R. \& Akogbeto M.C. 2015. Malaria vectors resistance to insecticides in Benin: current trends and mechanisms involved.Parasites \& Vectors 8.

27. Hamon J. 1963a. Les moustiques anthropophiles de la région de Bobo-Dioulasso (République de Haute-Volta): cycles d'agressivite et variations saisonnieres.In:Annales de la Societe entomologique de France, pp. 85-144.

28. Hamon J. 1963b. L'importance des changements de comportement chez les insectes.Bulletin of the World Health Organization 29: 115120.

29. Magesa S.M., Wilkes T.J., Mnzava A.E.P., Njunwa K.J., Myamba J., Kivuyo M.D.P.,Hill N., Lines J.D. \& Curtis C.F. 1991. Trial of pyrethroid impregnated bednets in an area of Tanzania holoendemic for malaria Part 2. Effects on the malaria vector population.Acta Tropica 49: 97-108.

30. Martinez-Torres D., Chandre F., Williamson M.S., Darriet F., Berge J.B., DevonshireA.L., Guillet P., Pasteur N. \& Pauron D. 1998. Molecular characterization of pyrethroid knockdown resistance $(\mathrm{kdr})$ in the major malaria vector Anopheles gambiae ss.Insect molecular biology 7: 179-184.

31. MS. 2013. Annuaire des statistiques sanitaires.Archive Ministère de la Santé, pp 87.

32. Müller P., Donnelly M.J. \& Ranson H. 2007. Transcription profiling of a recently colonised pyrethroid resistant Anopheles gambiae strain from Ghana.BMCgenomics 8: 36.

33. N'Guessan R., Vincent C., Martin A. et Mark R., 2007: Reduced Efficacy of Insecticide-treated Nets and Indoor Residual Spraying for Malaria Control in in Pyrethroid Resistance Area, Benin. Emerging Infectious Diseases.www.cdc.gov/eid.vol.13, ํ2, February 2007.

34. Nkya T.E., Akhouayri I., Poupardin R., Batengana B., Mosha F., Magesa S., Kisinza W. \& David J.-P. 2014. Insecticide resistance mechanisms associated with differentenvironments in the malaria vector Anopheles gambiae: a case study in Tanzania.Malaria Journal 13: 28 .

35. Organisation Mondiale de la Sante, 2004 : Lutte contre les vecteurs du paludisme. WHO/CDS/WHOPES/2002. 5Rev.1. 
36. Organisation Mondiale de la Sante1963: Méthode à suivre pour déterminer la sensibilité ou la résistance des larves de moustiques aux insecticides.In Résistance aux insecticides et lutte contre les vecteurs. Treizième rapport du comité OMS d'experts des insecticides, Genève : OMS, Sér. Rapp. Techn. 265, p. 55-60.

37. Poupardin R., Reynaud S., Strode C., Ranson H., Vontas J. \& David J.-P. 2008. Crossinductionof detoxification genes by environmental xenobiotics and insecticides inthe mosquito Aedes aegypti: Impact on larval tolerance to chemical insecticides.Insect biochemistry and molecular biology 38: 540-551.

38. Radhika W., Ankita R., Jasdeep K. S., Roopa S., Naim W., Sarita K., 2012: Larvicidal and irritant activities of hexane leaf extracts of Citrus sinensis against dengue vector Aedesaegypti L. Asian Pac. J. Trop. Biomed. 152-155.

39. Raghavendra K., Barik T.K. \& Adak T. 2010. Development of larval thermotoleranceand its impact on adult susceptibility to malathion insecticide and Plasmodiumvivax infection in Anopheles stephensi. Parasitology research 107: 1291-1297.

40. Ranson H., Jensen B., Vulule J.M., Wang X., Hemingway J. \& Collins F.H. 2000. Identification of a point mutation in the voltagegated sodium channel gene of Kenyan Anopheles gambiae associated with resistance to DDT and pyrethroids.Insect molecular biology 9: 491-497.

41. Roman Pavela., 2009: Larvicidal property of essential oils against Culexquinquefasciatus Say (Diptera: Culicidae). Industrial Crops and Products, 30(2), 311-315.

42. Sovi A., Azondékon R., Aïkpon R.Y., Govoétchan R., Tokponnon F., Agossa F., Salako A.S., Oké-Agbo F., Aholoukpè B., Okè M., Gbénou D., Massougbodji A. \& Akogbéto M. 2013. Impact of operational effectiveness of long-lasting insecticidal nets (LLINs) on malaria transmission in pyrethroid-resistant areas. Parasites \& Vectors 6: 319.

43. Traboulsi, A., El-Haj, S., Tueni, M., Taoubi, K., Nader, N.B., Mrad, A., 2005 : Repellency and toxicity of aromatic plant extracts against mosquito Culex pipiens molestus (Diptera:Culicidae). Pest.Manage.Sci. 61, 597-604.

44. Vulule J.M., Beach R.F., Atieli F.K., Roberts J.M., Mount D.L. \& Mwangi R.W. 1994.Reduced susceptibility of Anopheles gambiae to permethrin associated with the use of permethrin-impregnated bednets and curtains in Kenya. Medical and veterinaryentomology 8: 71-75. 
45. Yadouleton A., Martin T., Padonou G., Chandre F., Asidi A., Djogbénou L., Dabiré R., Aïkpon R., Boko M., Glitho I. \& others. 2011. Cotton pest management practices and the selection of pyrethroid resistance in Anopheles gambiae population in Northern Benin.Parasite \& Vectors 4: 1-11.

46. Yadouleton A.W., Padonou G., Asidi A., Moiroux N., Bio-Banganna S., Corbel V.,N'Guéssan R., Gbenou D., Yacoubou I., Gazard K. \&Akogbéto M.C. 2010. Insecticide resistance status in Anopheles gambiae in southern Benin. Malaria Journal 9: 83.

47. Yadouléton W.A., Padonou G.G., Assidi A., Nicolas M., Sahabib.B., Vincent C., Raphael N., Dina G., Imorou Y., Kinde G. et Martin A., 2010: Insecticide resistance status in Anopheles gambiae in Benin.Malar J 2010, 9:83. 